\title{
Effect of Vitamin D on Glucose Homeostasis, Sensitivity and Insulin Resistance in Type 2 Diabetes
}

\author{
Fatemeh Amirasgari ${ }^{1}$, Shaghayegh Torkfar ${ }^{1}$, Samaneh Rezazadeh ${ }^{1}$, Mohadeseh Farzaneh ${ }^{1}$ and Esra Tajik ${ }^{2 *}$ \\ ${ }^{1}$ Department of Medical Sciences, Islamic Azad University (IAUTMU), Iran \\ ${ }^{2}$ Faculty of Nutrition Sciences and Food Technology, Shahid Beheshti University of Medical Sciences, Iran
}

Submission: March 05, 2019; Published: July 09, 2019

*Corresponding author: Esra Tajik, Faculty of Nutrition Sciences and Food Technology, Shahid Beheshti University of Medical Sciences, P.O. Box: 1981619573, Tehran, Iran

\section{Abstract}

Background: In recent years, non-bone diseases have been reported with vitamin D deficiency, one of them is type 2 diabetes, which is a group of metabolic disorders caused by insulin action deficiency. Evidences suggest that vitamin D deficiency affects the metabolic function of cells, including beta-pancreatic cells. Regarding the fact that some studies have shown the effect of vitamin D on control of type 2 diabetes, the present study aims to review on the relationship between vitamin D, glucose homeostasis and insulin resistance type 2 diabetes.

Methods: This study is a review on descriptive and analytic studies which accessed data using biochemical tests, questionnaires and interviews. By using key words on vitamin D, type 2 diabetes and insulin resistance, 10 articles including descriptive-analytic, cross-sectional, case-control and interventional types from 1986 to 2018 were conducted.

Results: Many studies have shown that vitamin D levels of 25-hydroxyvitamin D in diabetics, women, and obese people are lower than others. It shows the effect of vitamin D on glucose homeostasis. In addition, vitamin D has an effect on intracellular calcium therefore it influences on insulin secretion. It can be said that vitamin D deficiency causes calcium inefficiency to regulate insulin secretion.

Conclusion: According to studies, it can be concluded that vitamin D might be effective in preventing type 2 diabetes and its controlling. Also, there is no association between vitamin D and insulin secreted by food and hemoglobin A1c (HbA1c).

Keywords: Vitamin D deficiencies; Diabetes mellitus; Type 2; Insulin secretion; Insulin resistance; Pancreatic gland; Fat; Protein; Carbohydrates; Steroid compounds; Ergocalciferol; Fungi; Yeast; Cholecalciferol

\section{Introduction}

Diabetes mellitus is a chronic disorder in pancreatic gland, which increases glucose levels due to low levels of insulin secretion or insufficiency of insulin activity in the body [1]. Type 2 diabetes leads to decreased insulin sensitivity and poor performance of pancreatic beta cells [2]. The consequence of disruption of insulin activity is disorder of fat, protein and carbohydrates [3].

Diabetes mellitus is the third leading cause of death in the world [4]. According to the World Health Organization (WHO), the number of diabetics has risen from 108 million in 1980 to 422 million in 2014 , with 1.5 million deaths directly due to diabetes in 2012 [5].

The statistics show that only $50 \%$ of the world's population has enough vitamin D [6]. The prevalence of vitamin D deficiencies varies according to gender, age and type of food intake, and the climate and skin color [7]. Decreased vitamin D levels are a factor in predicting the progression of type 2 diabetes [8]. This article is aimed to review the researches on the relationship between type 2 diabetes and vitamin D deficiency which vitamin D might be a preventive factor for type 2 diabetes.

\section{Vitamin D and its deficiency}

Looking again at vitamin D has led to a greater impact on cancer, cardiovascular disease, allergies and diabetes, and it's a source of attention for all [8]. Vitamin D is referred to as two types of steroid compounds: Ergocalciferol (D2) is produced by plants, more by fungi and yeast, and cholecalciferol (D3) is produced by humans by ultraviolet radiation on the skin. This form of vitamin D in fish oil is also available [8]. According to a 
study, exposure the face, hand, legs or back in front of sunlight for about 2 hours a day is likely to be enough to provide vitamin $\mathrm{D}$ [9]. This exposure is 3 minutes a day in the winter, 20 minutes in the autumn and 10 minutes in the summer, to let sunlight to provide vitamin D [10]. Food sources of vitamin D are also limited to salmon, lamb liver, and egg yolk [11].

Regarding the metabolism of vitamin D, the 25-hydroxylase enzyme in the liver and intestinal microsomes catalyzes the first step of vitamin D activating [9]. This process is so efficient that with only a small amount of vitamin D in the small intestine or liver, small amounts of pro-vitamin D can be found in the bloodstream [9]. Activation of 1.25-hydroxyvitamin D in the epithelium of the proximal tubular is also known as 1-monoxygenase 4, called 1-alpha-hydroxylase (a cytochrome P450 mitochondrial oxidase) [9].

Looking again at vitamin D has led to a greater impact on cancer, cardiovascular disease, and allergies and diabetes, and overall attention on it [8]. Due to the type of coverage among women in the Middle East, the lack of vitamin D in these areas is more severe than in Europe and the United States [12], however, vitamin D deficiency is common in women [13]. Since vitamin $\mathrm{D}$ is a fat solution and can be surrounded by fat molecules, overweight people are more likely to be exposed to Vitamin D deficiency [14]. Vitamin D deficiency is associated with atherosclerosis, depression, cancer, infectious and inflammatory bowel disease, migraine and multiple sclerosis [11].

\section{Epidemiology and pathology of type 2 diabetes}

The prevalence of type 2 diabetes is growing at an alarming rate in the world. Every year, more than a million people are diagnosed as new in the United States [15]. Recently, the prevalence of type 2 diabetes increased and genetic factors as well as environmental factors are associated with the onset of this disease [16]. Changes in the lifestyle including type of diet, physical activity, smoking, consumption of high-fat foods and reduced consumption of fruits and vegetables, have increased the prevalence of type 2 diabetes [17].

In addition to the genetic evaluation, some major factors related to diabetes are hormones, molecular and biochemical factors [18]. Increasing levels of triglycerides and cholesterol and lowering high density lipoprotein (HDL) in diabetics increases the risk of atherosclerosis [19]. Type 2 diabetes is most commonly seen in adults, which can be attributed to increased insulin resistance, overweight and low physical activity [17]. The higher prevalence of type 2 diabetes in most societies is seen as a result of lifestyle changes from traditional to industrial [13].

\section{The relationship between vitamin $D$ deficiency and type 2 diabetes}

The common characteristic between vitamin D deficiency and diabetes is that both of these are endemic diseases [8].8 Vitamin D deficiency and diabetes can be linked to osteoporosis and metabolic syndromes [13]. Common risk factors among them can be African American race, obesity, aging and reduced physical activity [13]. Also, old men with vitamin D deficiency release more insulin after absorption of glucose [13]. Recently, researchers have found that serum levels of 25-hydroxy vitamin D are considered to be an effective preventative factor for longterm diabetes mellitus complications including cardiovascular and renal diseases [20]. So, it can be concluded that diabetic patients with liver and/or renal diseases have a lower vitamin D deficiency [8]. It has been observed that 25-hydroxy vitamin D decreased in cases of type 2 diabetes, pregnancy diabetes and obesity. It has also been shown that insulinogenic indexes have improved with vitamin D supplementary.

On the other hand, the role of vitamin D on systemic inflammation can be pointed here briefly. Systemic inflammation has been linked initially with insulin resistance [15]. It can be concluded that it is also associated with type 2 diabetes [15]. Systemic inflammation of cytokines causes apoptosis in beta pancreatic cells [15]. It can say vitamin D can improve the survival of beta cells by modulating the effects of cytokines [15]. It also has an anti-inflammatory effect on reducing insulin resistance.

\section{Calcium, vitamin $\mathrm{D}$ and diabetes}

Indirect effect of calcium on insulin secretion is that vitamin D can maintain extracellular calcium density in normal level and hold calcium flux within the cell membrane at the natural level [8]. The effect of vitamin D deficiency on intracellular calcium is that if intracellular calcium increases, post-receptor binding insulin function is impaired, such as dephosphorylation of glycogen synthase and adjustable glucose transduction (GLUT4) [21].

Since insulin secretion from pancreatic $\beta$ cells associated with the amount of intracellular calcium and elevated intracellular calcium which inhibits insulin receptors, so lack of vitamin D can increase amount of parathyroid hormone and consequently, elevated intracellular calcium can inhibit insulin receptors [22]. Acute increase of intracellular calcium causes insulin target cells to not measure intracellular calcium flux for insulin performance [21]. Increased intracellular calcium can weaken beta-pancreatic cells [21]. The mentioned fact about vitamin D deficiency is not explicitly discussed [21]. It is also stated that the level of vitamin D does not correlated with basal insulin resistance in blood, but it significantly stimulates secretion of insulin after taking glucose [23].

In general, it can be concluded that, calcium loses its ability to affect insulin secretion in vitamin D deficiency [8]. In summary, vitamin D shows some improvements in the association of diabetes in actions such as stimulating the expression of the receptor of insulin as a result of increased insulin activity, indirect effect on calcium as a result of improved insulin secretion, and improvement the responsiveness of cells to transfer glucose into them [8]. 


\section{Design and Method}

This study is a review on descriptive, cross-sectional, interventional and analytical studies. By searching in Pubmed, Biomed central, SID and Iran doc, at the first step the key words of type 2 diabetes, insulin and vitamin $\mathrm{D}$ and at the next step, insulin resistance, insulin sensitivity, pregnancy and fasting blood sugar were searched. A total of 10 full text articles in English and Persian in period of 30 years (from 1986 to 2018) were selected.

Selection of articles preferably interventional, crosssectional and case-control studies was considered according to their subjects and goals. Certainly, qualitative studies were not included in the study. Studies with population over 100 participants up to 400 were entered the current study, but due to the value of studies, some interventional and/or case-control studies were selected however they conducted less than 100 participants. Most of the articles were in line with the goal of our study, and articles on type 1 diabetes and the effect of calcium intake on diabetes were excluded.

\section{Results}

The following statements show some researches in this field. According to the results, the major combination of vitamin D is 25-hydroxy vitamin D3, which is the highest representative of the level of vitamin D in blood [24]. The following are the findings of some studies which stated the predictive role of serum 25-hydroxy vitamin D for glycemic status and insulin resistance. For instance, a cross-sectional study on 524 men and women (40-69 years old), baseline mean serum 25- hydroxy vitamin D was lower in women $(57.2 \mathrm{nmol} / \mathrm{l})$ than men $(64.5$ $\mathrm{nmol} / \mathrm{l}$ ). Baseline 25-hydroxy vitamin D was associated inversely with risk of fasting glucose and fasting insulin [25]. In a casecontrol study on 90 participants with type 2 diabetes and 90 healthy subjects in Diabetes Clinic in Kermanshah-Iran it was found that $82.2 \%$ of diabetics and $61.1 \%$ of healthy subjects had vitamin D deficiency and the obese subjects had $5.4 \mathrm{ng} / \mathrm{ml}$ lower vitamin D levels compared to those with normal body mass index (BMI). There was a significant difference $(\mathrm{p}<0.05)$ in the 25 -hydroxyvitamin D levels between patients (12.4 $\pm 6.1 \mathrm{nmol} / \mathrm{L})$ and in the healthy group $(20.7 \pm 18 \mathrm{nmol} / \mathrm{L}) .20$ Another crosssectional study in 2015 on 84 patients with diabetes in Golestan hospital, Iran showed the level of 25-hydroxyvitamin D in women was significantly lower than men and there was a relationship between waist-hip ratio (WHR) and fasting blood sugar and vitamin D levels, but there was no significant relationship between vitamin D and insulin, HbA1c and Homeostasis Model Assessment of Insulin Resistance )HOMA-IR( levels) [26].

Moreover, a study of descriptive-analytical in Ghazvin-Iran, in 2012, among 69 participants (one group: diabetic and treated with drug and another group non-diabetic) stated that the average serum levels of vitamin D3 in diabetics was significantly less than non-diabetics $(56.7 \pm 19.1$ vs. $35.5 \pm 16.6)$. Diabetics according to glycated hemoglobin (HbA1c) were separated into two groups with controlled and uncontrolled of blood sugar. There was no significant statistical difference between serum levels of vitamin D3 of controlled and uncontrolled blood sugar groups. Average serum levels of vitamin D3 in men were more than women.

Serum levels of vitamin D3 did not have significant relationship with level of HbA1c in diabetics. Out of 69, sixtytwo participants were had metabolic syndrome. In patients with metabolic syndrome the average serum levels of vitamin D3 in diabetics $(36.1 \pm 16.9 \mathrm{nmol} / \mathrm{L})$ was significantly less than nondiabetic ( $60.2 \pm 18.5 \mathrm{nmol} / \mathrm{L})$ [27]. An interventional study aimed to measure insulin sensitivity in 100 diabetic patients $(30-70$ years old) from Arak, Iran [13]. Patients were given 50,000 units of vitamin D3 for 2 months. In conclusion, the serum level of FPG from pre to post intervention reached $7.6 \pm 2.04 \mathrm{nmol} / \mathrm{L}$ to 7.27 $\pm 2.16 \mathrm{nmol} / \mathrm{L}$, respectively and HOMA-IR reduced from 3.57 \pm 3.18 to $2.89 \pm 3.28 .13$

In addition, the findings of overseas studies were in line with the current study to show the association between vitamin D deficiency and diabetes. An interventional study by Gedic and colleagues measured insulin secretion among 4 Turkish women (average 32.7 years old) with vitamin D deficiency. Determination of metabolic activity of insulin and glucose was conducted by OGTT method. Participants were given $2000 \mathrm{IU} / \mathrm{d}$ oral cholecalciferol for 6 months and increased insulinogenic and insulin levels were observed [21].

Another study by Upreti et al. [24] with randomized controlled trial design and 60 Indian patients with coexisting type 2 diabetes and hypovitaminosis D in 2012-13 was done. Patients were supplemented with vitamin D for 6 month and results showed significant decrease in mean $\mathrm{HbA1c}$ levels (7.29\% to $7.02 \%$; $\mathrm{P}=0.01$ ), mean fasting plasma glucose (FPG) (131.4 to $102.6 \mathrm{mg} / \mathrm{dl} ; \mathrm{P}=0.04$ ).

In a cross-sectional study carried out by Boucher, 44 adolescents living in East London, an average age of 44.9 years with a glucose intolerance problem and an average vitamin D serum $<27.5 \mathrm{nmol} / \mathrm{L}$ participated. Insulin secretion was determined by the oral glucose tolerance test (OGTT). It was concluded that vitamin D had positive association with insulin and post-challenge $C$ peptide [21].

\section{Discussion}

In this study, it is tried to find an association between vitamin $\mathrm{D}$ and type 2 diabetes such as effect of vitamin $\mathrm{D}$ on insulin secretion, sensitivity and resistance. Lack of vitamin D can reduce insulin secretion and its supplementary in animals can save secreted insulin [8]. Pittas \& colleagues [15] explained evidences of vitamin D effects on glucose homeostasis including: a- existence of Vitamin D Dedicated Receptors (VDRs) on $\beta$ cells of pancreas.21 b- expression of 1-alphahydroxylase 


\section{Current Research in Diabetes \& Obesity Journal}

enzyme in $\beta$ cells that transforming 25-hydroxyvitamin $\mathrm{D}$ to 1.25-hydroxyvitamin D. c- existence of VDR on muscle cells. d- direct effect of 1.25-hydroxyvitamin $\mathrm{D}$ on transcription of insulin receptor gene. e- finally, increase of glucose transferring by insulin in vitro.

The effect of vitamin D on the reduction of chance of developing type 2 diabetes was investigated in Nurses Health Study and Mitri, even in another study, this decline was 55\% $[8,28]$. There was an inverse relationship between vitamin D deficiency and prevalence of type 2 diabetes in the study of National Health and Nutrition Examination Survey (NHANES III) in years between 1988 and 1994 [8]. According to NHANES study, the effect of race presented that there is a reverse relationship between the levels of vitamin $\mathrm{D}$ and the number of diabetic patients in whites, but no relationship has been reported in black people [4]

Studies have shown that, giving 25-hydroxyvitamin D increases insulin sensitivity up to $54 \%$ and its deficiency leads to increased insulin resistance [29]. Gunal $\mathrm{Al}$ et al. [30], stated that vitamin D3 increased the insulin sensitivity directly in patients who has a kidney disorder and subsequently hyperparathyroidism. Talaei [13] was observed only a concentration of 100-150 nmol/L of vitamin D can have a dramatic effect on insulin sensitivity.

Contrary, despite the positive effects of vitamin D, studies have found that efficacy of vitamin $D$ has been inverted in diabetic patients, for instance, in a study by Taylor [31], it has been observed that, high dose of vitamin $\mathrm{D}$ has been increased insulin resistance. This study only responded to 3 cases of vitamin D deficiency and non-insulin dependent diabetes [31]. Although vitamin D injection resulted in a significant increase in vitamin $\mathrm{D}$ levels in diabetics, none of them reached normal levels of vitamin D [6]. In the study of MC Gill, no association was found between $\mathrm{HbA1c}$ and vitamin $\mathrm{D}$, but it was found in Kositsawat study [21]. The differences reported in results are due to the demographic conditions of under study groups and use of experimental methods with various designs to measure insulin secretion and sensitivity [24]. In a study by Orwoll, there was no significant association between vitamin $\mathrm{D}$ and insulin that secreted by meal in diabetic patients, this is probably due to the fact that vitamin D cannot be effective for uncontrolled diabetes [21]. In interventional studies, due to different designs of methodology, such as different doses of vitamin D and the selection of specific racial groups, such as Caucasian, leads to lack of integrity in findings [21].

There are limitations in some of the cross-sectional studies, for instance, in Scragg and colleagues study [32] they were not sure if vitamin D levels affects glucose metabolism or vice versa. One of the strengths of this review was presenting the role of vitamin D in glucose homeostasis as well as insulin resistance. Vitamin D is usually ignored while its role is crucial in type 2 diabetes. It is suggested for future studies to show the role of other contributing factors, such as weight, BMI and calcium intake in correlation to type 2 diabetes.

\section{Conclusion}

There is no consensus on the efficacy of vitamin D in the improvement of diabetic patients and findings are different. In general, the results of studies show that vitamin D supplementation can reduce insulin resistance in patients with type 2 diabetes and also improve beta cells function. Also, vitamin D deficiency affects insulin response. Due to global vitamin D deficiency, vitamin D levels in type 2 diabetes should be considered and if necessary, vitamin D supplementary should be prescribed to improve blood sugar controlling and insulin sensitivity.

\section{Acknowledgement}

Thanks to the friends who helped us with the collection of articles.

\section{References}

1. Hosseini SA, Nemati J, Ranjbar M, Edalatmanesh MA, Zar A (2017) Hypoglycemic interactive effects of Ginger extract and endurance training in diabetic rats. Horizon Med Sci 23: 325-330.

2. Eftekhari MH, Akbarzadeh M, Dabbaghmansheh MH, Hasanzadeh J (2011) Effects of treatment with oral calcitriol on plasma glucose and insulin levels in noninsulin dependent diabetes mellitus patients. NSFT 6: $1-10$

3. Hosseini SE, Tavakoli F, Karami M (2014) Medicinal plants and treatment of diabetes mellitus. Clin Exc 2: 64-89.

4. Zamanpour L, Banitalebi E, Amirhosseini SE (2016) Effect of aerobic training on inflammatory markers and insulin resistance in women with type 2 diabetes mellitus. Iranian Journal of Diabetes and Metabolism 15: 300-311.

5. (2016) Global report on diabetes. World Health Organization. The Switzerland.

6. Bazyar N, Jafarian K, Shadman Z, Qorbani M, Khoshniatnikoo M (2014) Supplementation on improving vitamin D levels and insulin resistance in vitamin $\mathrm{D}$ insufficient or deficient type 2 diabetic. IJDLD 13: 425433.

7. Babaei M, Esmaeili Jadidi M, Heidari B, Gholinia H (2017) The relationship between vitamin $\mathrm{D}$ deficiency and non-specific shin pain in the elderly: A case control study. JBUMS 19: 15-20.

8. Martin T, Campbell RK (2011) Vitamin D and Diabetes. Diaspect 24: 113-118.

9. Cheraghpour M, Naghashian F, Ehrampush E, Davoodi SH, Mirzayrazaz J (2014) Investigating the protective effects of vitamin D on diabetes. JFUMS 4: 15-33.

10. Mottaghi T, Khoshravesh F, Askari G, Iraj B, Ghiasvand R (2013) Vitamin D and Migraine: Review of current evidence. J Isfahan Med Sch 31: $32-40$

11. Mozafari Khosravi H, Nabizadeh-Asl L, Yasini Ardakani SM, Hadinedoushan H, Barzegar K (2013) The effect of 2 different single injections of high dose of vitamin D on improving the depression in depressed patients with vitamin D deficiency: a randomized clinical trial. J Clin Psychopharmacol 33: 378-385. 


\section{Current Research in Diabetes \& Obesity Journal}

12. Saeednia A, Larijani B, Jalalinia S, Farzadfar F, Keshtkar A (2013) A survey on the prevalence of vitamin D deficiency in the Iranian population residing in the Islamic Republic of Iran over the period 1990-2010. IJDLD 12: 574-584.

13. Talaei A, Mohamadi M, Adgi Z (2013) The effect of vitamin D on insulin resistance in patients with type 2 diabetes. Diabetol Metab Syndr 5(1): 8.

14. Saremi A, Shavandi N, Shahrjerdi SH, Mahmoudi Z (2014) The effect of aerobic training with vitamin D supplementation on cardiovascular risk factors in obese women. JCT arak univ 4: 389-396.

15. Pittas AG, Lau J, Hu FB, Dawson-Houghes B (2007) The Role of Vitamin $\mathrm{D}$ and Calcium in Type 2 Diabetes. A Systematic Review and MetaAnalysis. JCEM 92(6): 2017-2029.

16. Rashidi H, Ghadirian SB, Shirinpour Z, Yazdanpanah L, Kaykhaei MA (2016) The effect of vitamin D supplementation on insulin resistance and glycemic control in patients with type 2 diabetes. IJPT 8: 11665 11674.

17. Fallah Hosseini H, Fakhrzadeh H, Larijani B, Sheikh Samani AH (2005) Review of medicinal plants used in diabetes. JMP 5: 1-8.

18. Hosseini SA, Zar A, Mansouri A (2016) Effect of Aloveraera with swimming training on Alanine Aminotransferase and Aspartate Aminotransferase levels of Diabetic Rats. NSFTJ 11: 29-38.

19.Zar A, Hosseini SA, Ahmadi F, Rezaei M (2016) Effects of Ginger together with swimming training on blood fat profile in adult diabetic rats with Streptozotocin. NSFTJ 11: 65-74.

20. Rahimi M, Izadi N, Niroumand E, Madani FR, Najafi F (2016) Comparison of vitamin D levels in patients with type 2 diabetes and healthy people in 2014. J Mashhad Univ Med Sci 59: 97-105.

21. Alvarez JA, Ashraf A (2010) Role of Vitamin D in Insulin Secretion and Insulin Sensitivity for Glucose Homeostasis. Int J Endocrinol.

22. Behradmanesh S, Roudini K, Baradaran A (2011) Effect of supplementary vitamin D on improvement glycemic parameters in patients with type 2 diabetic. J Isfahan Med Sch 29: 1157-1164.

23. Wagner H, Alvarsson M, Mannheimer B, Degerblad M, Östenson CG (2016) No effect of high-dose vitamin D treatment on $\beta$-cell function, insulin sensitivity, or glucose homeostasis in subjects with abnormal glucose tolerance: a randomized clinical trial. Diabetes Care 39(3) 345-352.
24. Upreti V, Maitri V, Dhull P, Handa A, Prakash MS, et al. (2018) Effect of oral vitamin D supplementation on glycemic control in patients with type 2 diabetes mellitus with coexisting hypovitaminosis D: A parallel group placebo controlled randomized controlled pilot study. Diabetes Metab Syndr 12(4): 509-512.

25. Forouhi NG, Luan J, Cooper A, Boucher BJ, Wareham NJ (2008) Baseline Serum 25-Hydroxy Vitamin D Is Predictive of Future Glycemic Status and Insulin Resistance, The Medical Research Council Ely Prospective Study 1990-2000. Diabetes 57(10): 2619-2625.

26. Pooraziz S, Haidari F, Karandish M, Zakerkish M, Arsang Jang S (2015) Assessment of the Serum Level of vitamin D and glycemic and anthropometric indices in patients with type 2 diabetes. Qom Univ Med Sci J 9: 49-56.

27. Ziaee A, Hashemipoor S, Karimzadeh T, Jalalpoor A, Javadi A (2012) Relation of vitamin D3 level with metabolic syndrome indices among patients with diabetes and non-diabetic individuals. J Ardabil Univ Med Sci 12: 149-156

28. Mitri J, Dawson-Hughes B, Hu FB, Pittas AG (2011) Effects of vitamin $D$ and calcium supplementation on pancreatic $\beta$ cell function, insulin sensitivity, and glycemia in adults at high risk of diabetes: Calcium and Vitamin D for Diabetes Mellitus (CaDDM) randomized controlled trial. Am J Clin Nutr 94(2): 486-494.

29. Talaei A, Mohamadi Kelishadi M, Adgi Z (2011) The evaluation of the effect of vitamin D on insulin resistance in type II diabetic patients. J Arak Uni Med Sci 14: 79-84.

30. Gunal AI, Celiker H, Celebi H, Ustündağ B, Günal SY (1997) Intravenous Alfacalcidol improves insulin resistance in hemodialysis patients. Clin Nephrol 48(2): 109-113.

31. Taylor AV, Wise PH (1998) Vitamin D replacement in Asians with diabetes may increase insulin resistance. Postgrad Med J 74(872): 365-366.

32. Scragg R, Sowers M, Bell C (2004) Serum 25-hydroxyvitamin D, diabetes and ethnicity in the third national health and nutrition examination survey. Diabetes Care 27(12): 2813-2818. 